\title{
Global Stability of HIV-1 Infection Model with Two Time Delays
}

\author{
Hui Miao, Xamxinur Abdurahman, and Ahmadjan Muhammadhaji \\ College of Mathematics and System Science, Xinjiang University, Xinjiang, Urumqi 830046, China \\ Correspondence should be addressed to Xamxinur Abdurahman; xamxinur@sina.com
}

Received 8 October 2013; Accepted 19 November 2013

Academic Editor: Ming Mei

Copyright (C) 2013 Hui Miao et al. This is an open access article distributed under the Creative Commons Attribution License, which permits unrestricted use, distribution, and reproduction in any medium, provided the original work is properly cited.

\begin{abstract}
We investigate global dynamics for a system of delay differential equations which describes a virus-immune interaction in vivo. The model has two time delays describing time needed for infection of cell and CTLs generation. Our model admits three possible equilibria: infection-free equilibrium, CTL-absent infection equilibrium, and CTL-present infection equilibrium. The effect of time delay on stability of the equilibria of the CTL immune response model has been studied.
\end{abstract}

\section{Introduction}

As well known, in recent years the population dynamics of infectious diseases have been extensively studied [1-20]. Particularly, the HIV (human immunodeficiency virus) has been extensively studied in $[1-5,7-11,14,15,20]$ and became a global problem. The HIV infection is characterized by three different phases, namely, the primary infection, clinically asymptomatic stage (chronic infection), and acquired immunodeficiency syndrome (AIDS) or drug therapy. During primary infection, viral load in the peripheral blood experiences a substantial increase to the peak level, followed by decline to the steady state, which is referred to as the viral set point. Extremely high viral load during primary infection leads to the activation of $\mathrm{CD}^{+} \mathrm{T}$ cells, which are recognized as cytotoxic T cells (CTL) capable of suppressing viral replication. Viral decline from the peak is due to the control by these immune cells and/or limited target cell availability. The viral set point has been shown to be predictive for the pace of disease development [8]. Clinical research combined with mathematical modeling has enhanced progress in the understanding of HIV-1 infection [4]. This is because mathematical models can offer a way to study the dynamics of viral load in vivo and can be very useful in understanding the interaction between virus and host cell.

On the other hand, in the real situation, there may be a lag between the time target cells are contacted by the virus particles and the time the contacted cells become actively affected meaning that the contacting virions enter cells. This can be explained by the initial (or eclipse) phase of the virus life cycle, which include all stages from viral attachment until the time that the host cell contains the infectious viral particles in its cytoplasm [3]. Research [8] has shown that models of HIV-1 infection that include intracellular delays are more accurate representations of the biology and change the estimated values of kinetic parameters when compared to models without delays. Therefore, we should introduce time delays into model foundation, which will have more resemblance to the real ecosystem. In the last decade, the HIV-infection models with time delay have been studied by many authors, and time delays of one type or another have been incorporated into biological models by many authors (e.g., $[1-5,8-11,14,15,20]$ and the references cited therein). Here we include an intracellular delay as well as immune delay. There are some models which include an intracellular delay [1-5]; some authors believe that time delays can not be ignored in models for immune response $[6,7]$.

The salient features of the mechanism of the immune response during viral infection are as follows. First, the free virus enters its target, a susceptible cell. Inside this cell it replicates itself. And this susceptible cell becomes an infected cell. Then the infected cell dies and releases new viruses; these viruses begin to infect other susceptible cells. During the process of viral infection, the host is induced which is initially rapid and nonspecific (natural killer cells, macrophage cell, etc.) and then delayed and specific (cytotoxic T lymphocyte cells, antibody cell). But in most virus infections cytotoxic $\mathrm{T}$ 
lymphocyte (CTL) cells which attack infected cells, and antibody cell which attack viruses, play a critical part in antiviral defense. In order to investigate the role of the population dynamics of viral infection with CTL response, Pawelek et al. [8] constructed a mathematical model describing the basic dynamics of the interaction between the uninfected target cells, productively infected cells, free virus, and the CTL response cells, which is described by the following differential equation:

$$
\begin{gathered}
\frac{d x}{d t}=s-d x(t)-\beta x(t) v(t) \\
\frac{d y}{d t}=\beta e^{-m \tau_{1}} x\left(t-\tau_{1}\right) v\left(t-\tau_{1}\right)-a y(t)-p y(t) z(t), \\
\frac{d v}{d t}=k y(t)-u v(t) \\
\frac{d z}{d t}=c y\left(t-\tau_{2}\right)-b z(t)
\end{gathered}
$$

where the uninfected target cells are denoted by $x(t)$, productively infected cells are denoted by $y(t)$, free virus is denoted by $v(t)$, and the CTL response cells are denoted by $z(t)$. The parameter $s$ represents the rate at which new target cells are created, $d$ is the death rate of uninfected target cells, $\beta$ is the infection rate of uninfected cells by virus, $a$ is the death rate of productively infected cells, $p$ represents the killing rate of infected cells by CTL response cells, $k$ is the rate of the virus particles produced by infected cells, $u$ is the viral clearance rate constant, $c$ is the rate at which the CTL response is produced, and $b$ is the death rate of the CTL response.

In order to incorporate the intracellular phase of the virus life cycle, we assume that virus production occurs after the virus entry by the constant delay $\tau_{1}$. The recruitment of virus-producing cells at time $t$ is given by the number of the uninfected $\mathrm{CD}^{+} \mathrm{T}$ cells that were newly infected at time $t-\tau_{1}$ and are still alive at time $t[9,10]$. If we assume constant death rates $m$ for infected $\mathrm{CD} 4^{+} \mathrm{T}$ cells but not yet producing virus particles, the probability of surviving the time period from $t-\tau_{1}$ to $t$ is $\exp ^{-m \tau_{1}}$. And the immune response plays an important role in eliminating or controlling the disease after human body is infected by virus. Antigenic stimulation generating CTLs may need a period of time $\tau_{2}$; that is, the CTL response at time $t$ may depend on the population of antigen at a period time $t-\tau_{2}[11]$.

Therefore, based on the discussion above, the model can be written in the following form:

$$
\begin{gathered}
\frac{d x}{d t}=s-d x(t)-\beta x(t) v(t) \\
\frac{d y}{d t}=\beta e^{-m \tau_{1}} x\left(t-\tau_{1}\right) v\left(t-\tau_{1}\right)-a y(t)-p y(t) z(t), \\
\frac{d v}{d t}=k y(t)-u v(t) \\
\frac{d z}{d t}=c y\left(t-\tau_{2}\right) z\left(t-\tau_{2}\right)-b z(t) .
\end{gathered}
$$

The organization of this paper is as follows. In the next section we deal with some basic properties such as positivity and boundedness of the solutions and existence of equilibria of system (2). In Section 3, we prove the local stability of three possible equilibria. Further, by using the well-known Lyapunov-Lasalle invariance principle, we prove the global asymptotic stability of the infection-free equilibrium, CTLabsent infection equilibrium, and a special case of CTLpresent equilibrium. By bifurcation theory, we also can prove that there is a stability switch for another special case for CTL-present equilibrium. In Section 4, one example is given to illustrate that our main results are applicable. In the final section, we offer a brief conclusion.

\section{Basic Results}

Based on the biological meaning, we will consider the system (2) with the following initial conditions:

$$
\begin{gathered}
x(\theta) \geq 0, \quad y(\theta) \geq 0, \quad v(\theta) \geq 0, \quad z(\theta) \geq 0, \quad \theta \in[-\tau, 0] \\
x(0)>0, \quad y(0)>0, \quad v(0)>0, \quad z(0)>0 .
\end{gathered}
$$

Let $X=C([-\tau, 0] ; R)$ be the Banach space of continuous functions mapping the internal $[-\tau, 0]$ into $R_{+}^{4}$ equipped with the sup-norm, where $\tau=\max \left\{\tau_{1}, \tau_{2}\right\}$. Based on the existence and uniqueness theory of solution for functional differential equations $[12,13]$, it is easy to show that there is a unique solution $(x(t), y(t), v(t), z(t))$ to system (2) with initial condition (3).

First we will discuss the positivity and boundedness of the solution.

Theorem 1. Let $((x(t), y(t), v(t), z(t))$ be the solution of (2) satisfying conditions (3); then $x(t), y(t), v(t)$, and $z(t)$ are positive and ultimately bounded.

Proof. From (2), we have

$$
\begin{gathered}
x(t)=x(0) e^{-\int_{0}^{t}(d+\beta v(\xi)) \mathrm{d} \xi}+\int_{0}^{t} s e^{-\int_{\eta}^{t}(d+\beta v(\xi)) \mathrm{d} \xi} \mathrm{d} \eta, \\
y(t)=y(0) e^{-\int_{0}^{t}(a+p z(\xi)) \mathrm{d} \xi} \\
+\int_{0}^{t} \beta e^{-m \tau_{1}} v\left(t-\tau_{1}\right) x\left(t-\tau_{1}\right) e^{-\int_{\eta}^{t}(a+p z(\xi)) \mathrm{d} \xi} \mathrm{d} \eta, \\
v(t)=v(0) e^{-u t}+\int_{0}^{t} k y(\eta) e^{-u(t-\eta)} \mathrm{d} \eta, \\
z(t)=z(0) e^{-b t}+\int_{0}^{t} c y\left(\eta-\tau_{2}\right) z\left(\eta-\tau_{2}\right) e^{-b(t-\eta)} \mathrm{d} \eta .
\end{gathered}
$$

This means that all the solutions of system (2) with initial condition (3) are positive. To prove the boundedness of the solution, denote

$$
N(t)=e^{-m \tau_{1}} x\left(t-\tau_{1}\right)+y(t)+\frac{a}{2 k} v(t)+\frac{p}{c} z\left(t+\tau_{2}\right) .
$$


Calculating the derivative of $N(t)$ along the solution of system (2) and by positivity of the solutions, we have

$$
\begin{aligned}
\dot{N}(t)= & e^{-m \tau_{1}} s-e^{-m \tau_{1}} d x\left(t-\tau_{1}\right) \\
& -\frac{1}{2} a y(t)-\frac{a u}{2 k} v(t)-\frac{p b}{c} z\left(t+\tau_{2}\right) \\
\leq & e^{-m \tau_{1}} s-q N(t)
\end{aligned}
$$

where $q=\min \{d, a / 2, u, b\}$. This implies that $N(t)$ is bounded for large $t$. So $x(t), y(t), v(t)$, and $z(t)$ are ultimately bounded.

Next we will discuss the equilibria of system (2). The following is the basic reproductive ratio of system (2) which describes the average number of newly infected cells generated from one infected cell at the beginning of the infectious process:

$$
R_{0}=\frac{k \beta s e^{-m \tau_{1}}}{a d u}
$$

By direct calculation we have that system (2) has three equilibria. Infection-free equilibrium $E_{0}=\left(x_{0}, 0,0,0\right)=$ $(s / d, 0,0,0)$. If $R_{0}>1$, there is a CTL-absent infection equilibrium

$$
\begin{aligned}
E_{1} & =(\bar{x}, \bar{y}, \bar{v}, 0) \\
& =\left(\frac{a u e^{m \tau_{1}}}{\beta k}, \frac{\beta k s e^{-m \tau_{1}}-a d u}{k a \beta}, \frac{\beta k s e^{-m \tau_{1}}-a d u}{u a \beta}, 0\right) .
\end{aligned}
$$

If $R_{0}>1+\beta k b / u c d$, then there is a CTL-present infection equilibrium

$$
\begin{aligned}
E_{2} & =\left(x^{*}, y^{*}, v^{*}, z^{*}\right) \\
& =\left(\frac{s u c}{\beta k b+u c d}, \frac{b}{c}, \frac{k b}{u c}, \frac{\beta k s c e^{-m \tau_{1}}-a(c d u+k b \beta)}{p(c d u+k b \beta)}\right) .
\end{aligned}
$$

\section{Stability Analysis of Delay Model (2)}

In this section, we will analyse locally and globally asymptotic stability of the three equilibria.

\subsection{Stability of Infection-Free Equilibrium $E_{0}$}

Theorem 2. For the infection-free equilibrium $E_{0}$ of system (2);

(i) if $R_{0}<1$, then $E_{0}$ is locally asymptotically stable;

(ii) if $R_{0}>1$, then $E_{0}$ is unstable.

Proof. First, we will prove the local stability of $E_{0}$. At the infection-free equilibrium $E_{0}$, the characteristic equation for the corresponding linearized system of (2) becomes

$$
(\lambda+b)(\lambda+d)\left[(\lambda+a)(\lambda+u)-\beta k \frac{s}{d} e^{-(m+\lambda) \tau_{1}}\right]=0 .
$$

Two of the roots of the characteristic equation (10) are $\lambda_{1}=$ $-b$, and $\lambda_{2}=-d$. The remaining two roots are obtained by considering the following equation:

$$
(\lambda+a)(\lambda+u)=\beta k \frac{s}{d} e^{-(m+\lambda) \tau_{1}}
$$

If $\lambda$ has a nonnegative real part, then the modulus of the lefthand side of (11) satisfies

$$
|(\lambda+a)(\lambda+u)| \geq a u
$$

while the modulus of the right-hand side (11) satisfies

$$
\beta k \frac{s}{d} e^{-(m+\lambda) \tau_{1}}=\left|a u e^{-\lambda \tau_{1}} R_{0}\right| \leq a u R_{0}<a u .
$$

This leads to a contradiction. Thus, when $R_{0}<1$, all the eigenvalues have negative real parts, and hence the infectionfree steady state is locally asymptotically stable.

When $R_{0}>1$, let

$$
\begin{aligned}
f(\lambda) & =(\lambda+a)(\lambda+u)-\beta k \frac{s}{d} e^{-(m+\lambda) \tau_{1}} \\
& =(\lambda+a)(\lambda+u)-a u e^{-\lambda \tau_{1}} R_{0}
\end{aligned}
$$

Then $f(0)=a u-a u R_{0}<0, \lim _{\lambda \rightarrow+\infty} f(\lambda)=+\infty$. By the continuity of $f(\lambda)$, there exists at least one positive root of $f(\lambda)=0$. Thus, the infection-free equilibrium $E_{0}$ is unstable if $R_{0}>1$.

Theorem 3. If $R_{0}<1$, then infection-free steady state $E_{0}$ is globally asymptotically stable.

Proof. Define a Lyapunov functional

$$
\begin{aligned}
V(t)= & x_{0}\left(\frac{x}{x_{0}}-\ln \frac{x}{x_{0}}-1\right)+e^{m \tau_{1}} y+\frac{\beta s}{u d} v+\frac{p e^{m \tau_{1}}}{c} z \\
& +\beta \int_{t-\tau_{1}}^{t} v(s) x(s) \mathrm{d} s+p e^{m \tau_{1}} \int_{t-\tau_{2}}^{t} y(s) z(s) \mathrm{d} s,
\end{aligned}
$$

where $x_{0}=d / s$. Calculating the time derivative of $V(t)$ along the solution of system (2), we obtain

$$
\begin{aligned}
\left.D^{+} V\right|_{(2)}= & \left(1-\frac{x_{0}}{x}\right)\left(d x_{0}-d x(t)-\beta x(t) v(t)\right) \\
& +e^{m \tau_{1}}\left[\beta e^{-m \tau_{1}} x\left(t-\tau_{1}\right) v\left(t-\tau_{1}\right)\right.
\end{aligned}
$$




$$
\begin{gathered}
-a y(t)-p y(t) z(t)] \\
+\frac{\beta s}{u d}(k y(t)-u v(t)) \\
+\frac{p e^{m \tau_{1}}}{c}\left[c y\left(t-\tau_{2}\right) z\left(t-\tau_{2}\right)-b z(t)\right] \\
+\beta x(t) v(t)-\beta x\left(t-\tau_{1}\right) v\left(t-\tau_{1}\right) \\
+p e^{m \tau_{1}} y(t) z(t)-p e^{m \tau_{1}} y\left(t-\tau_{2}\right) z\left(t-\tau_{2}\right) \\
=-d \frac{1}{x}\left(x-x_{0}\right)^{2} \\
-\left(a e^{m \tau_{1}}+\frac{k \beta s}{u d}\right) k y(t)-\frac{p e^{m \tau_{1}}}{c} b z(t) \leq 0 .
\end{gathered}
$$

Note that $\left.D^{+} V(t)\right|_{(2)}=0$ if and only if $x=x_{0}, y(t)=$ $0, z(t)=0$. By the second equation of (2), we also have $v(t)=0$. Therefore, the maximal compact invariant set in $\left.D^{+} V(t)\right|_{(2)}=0$ is the singleton $E_{0}$. By the Lasalle Invariance principle [12], the infection-free steady state $E_{0}$ is globally attracting. Therefore, $E_{0}$ is globally asymptotically stable.

\subsection{Stability of the CTL-Absent Infection Equilibrium $E_{1}$}

Theorem 4. For the CTL-absent infection equilibrium $E_{1}$ of system (2),

(i) if $1<R_{0}<1+\beta k b /$ ucd, then $E_{1}$ is locally asymptotically stable;

(ii) if $R_{0}>1+\beta k b / u c d$, then $E_{1}$ is unstable.

Proof. At the CTL-absent infection equilibrium $E_{1}$, the characteristic equation for the corresponding linearized system of (2) is

$$
\begin{aligned}
& \left(\lambda-c \bar{y} e^{-\lambda \tau_{2}}+b\right) \\
& \quad \times\left[\lambda^{3}+a_{1} \lambda^{2}+a_{2} \lambda+a_{3}+\left(a_{4} \lambda+a_{5}\right) e^{-\lambda \tau_{1}}\right]=0,
\end{aligned}
$$

where

$$
\begin{aligned}
a_{1} & =d+\beta \bar{v}+a+u=d+\frac{\beta k s e^{-m \tau_{1}}-a u d}{a u}+a+u, \\
a_{2} & =a u+(a+u)(d+\beta \bar{v}) \\
& =a u+(a+u)\left(d+\frac{\beta k s e^{-m \tau_{1}}-a u d}{a u}\right), \\
a_{3} & =a u(d+\beta \bar{v})=a u\left(d+\frac{\beta k s e^{-m \tau_{1}}-a u d}{a u}\right), \\
a_{4} & =-k e^{-m \tau_{1}} \beta \bar{x}, \\
a_{5} & =-k d e^{-m \tau_{1}} \beta \bar{x} .
\end{aligned}
$$

First we consider

$$
\lambda^{3}+a_{1} \lambda^{2}+a_{2} \lambda+a_{3}+\left(a_{4} \lambda+a_{5}\right) e^{-\lambda \tau_{1}}=0 .
$$

When $\tau_{1}=0$, (19) becomes

$$
\lambda^{3}+a_{1} \lambda^{2}+\left(a_{2}+a_{4}\right) \lambda+\left(a_{3}+a_{5}\right)=0 .
$$

As $a_{1}=a+u>0, a_{2}+a_{4}=(a+u)(d+(\beta k s-a u d) / a u)>0$, $a_{3}+a_{5}=\beta k s-a d u>0$, by Routh-Hurwitz criterion, we only need to show the following statement:

$$
\begin{aligned}
a_{1} & \left(a_{2}+a_{4}\right)-\left(a_{3}+a_{5}\right) \\
& =\left(\frac{\beta k s}{a u}+a+u\right)(a+u) \frac{\beta k s}{a u}-k \beta s+a d u>0 .
\end{aligned}
$$

Thus all the roots of (19) have negative real parts when $\tau_{1}=0$.

Now we consider the distribution of roots of (19) while $\tau_{1} \neq 0$. If $i w(w>0)$ is a solution of (19), separating real and imaginary parts, it follows that

$$
\begin{aligned}
& a_{1} w^{2}-a_{3}=a_{4} w \sin \left(w \tau_{1}\right)+a_{5} \cos \left(w \tau_{1}\right), \\
& w^{3}-a_{2} w=a_{4} w \cos \left(w \tau_{1}\right)-a_{5} \sin \left(w \tau_{1}\right) .
\end{aligned}
$$

Squaring and adding the two equations of (22) yield

$$
\begin{aligned}
w^{6}+ & \left(a_{1}^{2}-2 a_{2}\right) w^{4} \\
& +\left(a_{2}^{2}-2 a_{1} a_{3}-a_{4}^{2}\right) w^{2}+a_{3}^{2}-a_{5}^{2}=0 .
\end{aligned}
$$

Letting $r=w^{2}$, (23) becomes

$$
r^{3}+\left(a_{1}^{2}-2 a_{2}\right) r^{2}+\left(a_{2}^{2}-2 a_{1} a_{3}-a_{4}^{2}\right) r+a_{3}^{2}-a_{5}^{2}=0,
$$

where

$$
\begin{gathered}
a_{1}^{2}-2 a_{2}=(d+\beta \bar{v})^{2}+\left(a^{2}+u^{2}\right)>0, \\
a_{2}^{2}-2 a_{1} a_{3}-a_{4}^{2}=\left(a^{2}+u^{2}\right)(d+\beta \bar{v})^{2}>0, \\
a_{3}^{2}-a_{5}^{2}=[a u \beta \bar{v}],[a u(2 d+\beta \bar{v})]>0, \\
\left(a_{1}^{2}-2 a_{2}\right)\left(a_{2}^{2}-2 a_{1} a_{3}-a_{4}^{2}\right)-\left(a_{3}^{2}-a_{5}^{2}\right) \\
=\left(a^{2}+u^{2}\right)(d+\beta \bar{v})^{4}+\left(a^{4}+u^{4}\right)[d+\beta \bar{v}]^{2} \\
+2 a^{2} u^{2} d^{2}+a^{2} u^{2}(\beta \bar{v})^{2}+2 a^{2} u^{2} d \beta \bar{v}>0 .
\end{gathered}
$$

By the Routh-Hurwitz criterion, (24) has no positive roots. This shows that (19) can not have a purely imaginary root.

Next, we analyze the transcendental equation

$$
\lambda-c \bar{y} e^{-\lambda \tau_{2}}+b=0 .
$$

For $\tau_{2}=0$, if $1<R_{0}<1+\beta k b / u c d$, we have $\lambda=c \bar{y}-b=$ $(\beta k s c-a \beta k b-a c u d) / a \beta k<0$. This shows that the root of (26) is negative for $\tau_{2}=0$.

Now we only need to consider (26) in the case $\tau_{2}>0$. By letting $\lambda=\omega i(\omega>0)$ be a purely imaginary root of (26) for some $\omega>0$, we have

$$
\begin{gathered}
\omega=-\frac{c\left(\beta k s e^{-m \tau_{1}}-a u d\right)}{a k \beta} \sin \omega \tau_{2}, \\
b=\frac{c\left(\beta k s e^{-m \tau_{1}}-a u d\right)}{a k \beta} \cos \omega \tau_{2},
\end{gathered}
$$


which implies that $\omega^{2}=\left[c\left(\beta k s e^{-m \tau_{1}}-a u d\right) / a k \beta\right]^{2}-b^{2}$. Note that $1<R_{0}<1+\beta k b / u c d$; then $\omega^{2}<0$, which is a contradiction.

Therefore, we conclude that the characteristic equation (17) does not have any root with nonnegative real part. By the general theory of delay differential equations from Kuang [12], we see that if $1<R_{0}<1+\beta k b / u c d$, the equilibrium $E_{1}$ is locally asymptotically stable.

Theorem 5. If $1<R_{0}<1+\beta k b / u c d$, then the CTL-absent infection equilibrium $E_{1}$ of system (2) is globally asymptotically stable.

Proof. Denote $g(\xi)=\xi-1-\ln \xi$, $\xi \in R^{+}$. Define a Lyapunov functional

$$
\begin{aligned}
V(t)= & \bar{x} g\left(\frac{x}{\bar{x}}\right)+e^{m \tau_{1}} \bar{y} g\left(\frac{y}{\bar{y}}\right)+\frac{a e^{m \tau_{1}}}{k} \bar{v} g\left(\frac{v}{\bar{v}}\right) \\
& +\frac{p e^{m \tau_{1}}}{c} \bar{z} g\left(\frac{z}{\bar{z}}\right)+\beta \bar{v} \bar{x} \int_{t-\tau_{1}}^{t} g\left(\frac{x(s) v(s)}{\bar{v} \bar{x}}\right) \mathrm{d} s \\
& +p e^{m \tau_{1}} \int_{t-\tau_{2}}^{t} y(s) z(s) \mathrm{d} s .
\end{aligned}
$$

By calculating the derivative of $V(t)$ along the solution of system (2), we obtain that

$$
\begin{aligned}
D^{+} V & \left.(t)\right|_{(2)} \\
= & \dot{x}(t)\left(1-\frac{\bar{x}}{x}\right)+e^{m \tau_{1}} \dot{y}(t)\left(1-\frac{\bar{y}}{y}\right) \\
& +\frac{a e^{m \tau_{1}}}{k} \dot{v}(t)\left(1-\frac{\bar{v}}{v}\right)+\frac{p e^{m \tau_{1}}}{c} \dot{z}(t)\left(1-\frac{\bar{z}}{z}\right) \\
& +\beta \bar{v} \bar{x}\left[g\left(\frac{v(t) x(t)}{\bar{v} \bar{x}}\right)-g\left(\frac{v\left(t-\tau_{1}\right) x\left(t-\tau_{1}\right)}{\bar{v} \bar{x}}\right)\right] \\
& +p e^{m \tau_{1}}\left[y(t) z(t)-y\left(t-\tau_{2}\right) z\left(t-\tau_{2}\right)\right] \\
= & \left(1-\frac{\bar{x}}{x}\right)[d \bar{x}+\beta \bar{v} \bar{x}-d x(t)-\beta x(t) v(t)] \\
& +e^{m \tau_{1}}\left(1-\frac{\bar{y}}{y}\right) \\
& +\left[\beta e^{-m \tau_{1}} x\left(t-\tau_{1}\right) v\left(t-\tau_{1}\right)-a y(t)-p y(t) z(t)\right] \\
& +\frac{a e^{m \tau_{1}}}{k}\left[1-\frac{\bar{v}}{v}\right](k y(t)-u v(t)) \\
& +\frac{p e^{m \tau_{1}}}{c}\left(1-\frac{\bar{z}}{z}\right)\left[c y\left(t-\tau_{2}\right) z\left(t-\tau_{2}\right)-b z(t)\right] \\
& +\beta x(t) v(t)-\beta x\left(t-\tau_{1}\right) v\left(t-\tau_{1}\right) \\
& +\beta \bar{v} \bar{x} \ln \frac{v(t) x(t)}{v\left(t-\tau_{1}\right) x\left(t-\tau_{1}\right)} \\
& +p e^{m \tau_{1}}\left[y(t) z(t)-y\left(t-\tau_{2}\right) z\left(t-\tau_{2}\right)\right] .
\end{aligned}
$$

Remember that

$$
s=d \bar{x}+\beta \bar{v} \bar{x}, \quad \beta e^{-m \tau_{1}} \bar{v} \bar{x}=a \bar{y}, \quad k \bar{y}=u \bar{v} .
$$

The equation above can be rewritten as

$$
\begin{aligned}
& \left.D^{+} V\right|_{(2)} \\
& =d \bar{x}\left(2-\frac{\bar{x}}{x}-\frac{x}{\bar{x}}\right) \\
& +\beta \bar{v} \bar{x}\left[3-\frac{\bar{x}}{x}-\frac{\bar{y} x\left(t-\tau_{1}\right) v\left(t-\tau_{1}\right)}{y \bar{x} \bar{v}}\right. \\
& \left.-\frac{y(t) \bar{v}}{v(t) \bar{y}}-\ln \frac{v(t) x(t)}{v\left(t-\tau_{1}\right) x\left(t-\tau_{1}\right)}\right] \\
& +p e^{m \tau_{1}} z(t)\left(\bar{y}-\frac{b}{c}\right) \\
& =d \bar{x}\left(2-\frac{\bar{x}}{x}-\frac{x}{\bar{x}}\right) \\
& -\beta \bar{v} \bar{x}\left[g\left(\frac{\bar{y} x\left(t-\tau_{1}\right) v\left(t-\tau_{1}\right)}{y \bar{x} \bar{v}}\right)\right. \\
& \left.+g\left(\frac{\bar{x}}{x}\right)+g\left(\frac{y(t) \bar{v}}{v(t) \bar{y}}\right)\right] \\
& +p e^{m \tau_{1}} z(t)\left(\bar{y}-\frac{b}{c}\right) .
\end{aligned}
$$

From (31), it follows that $\left.D^{+} V(t)\right|_{(2)} \leq 0$ for all $x, y, v, z>$ 0. $\left.D^{+} V(t)\right|_{(2)}=0$ if and only if $(x(t), y(t), v(t), z(t))=$ $(\bar{x}, \bar{y}, \bar{z}, 0)$. Then the globally asymptotic attractivity of $E_{1}$ follows from Lyapunov-Lasalle invariance principle [12]. Therefore, $E_{1}$ is globally asymptotically stable.

3.3. Stability of CTL-Present Infection Equilibrium $E_{2}$. On the stability analysis of CTL-present infection equilibrium $E_{2}$, we only consider the two special cases, that is, $\tau_{2}=0, \tau_{1} \neq 0$ and $\tau_{1}=0, \tau_{2} \neq 0$.

Firstly, we consider the local and global stability of $E_{2}$ for the first case, and we have the following results.

Theorem 6. If $\tau_{2}=0, \tau_{1} \neq 0$ and $R_{0}>1+\beta k b / u c d$, then the CTL-present infection equilibrium $E_{2}$ is locally asymptotically stable.

Proof. At equilibrium $E_{2}$, the characteristic equation for the corresponding linearized system of (2) is

$$
\begin{aligned}
(\lambda+ & \left.d+\beta v^{*}\right)(\lambda+u) \\
& \times\left[\left(\lambda+b-c y^{*} e^{-\lambda \tau_{2}}\right)\left(\lambda+\delta R_{0}\right)+b\left(\delta R_{0}-a\right) e^{-\lambda \tau_{2}}\right] \\
= & \left(\lambda+b-c y^{*} e^{-\lambda \tau_{2}}\right)(\lambda+d) u \delta R_{0} e^{-\lambda \tau_{1}},
\end{aligned}
$$


where $\delta=\operatorname{aucd} /(u c d+k b \beta)$. Further let $\tau_{2}=0$; then (32) becomes

$$
\begin{aligned}
\left(\lambda+d+\beta v^{*}\right)(\lambda+u)\left[\lambda\left(\lambda+\delta R_{0}\right)+b\left(R_{0} \delta-a\right)\right] \\
=\lambda(\lambda+d) u \delta R_{0} e^{-\lambda \tau_{1}}
\end{aligned}
$$

By the continuous dependence of roots of the characteristic equation on $R_{0}$, we know that the curve of the roots must cross the imaginary axis as $R_{0}$ decreases sufficiently close to 1 . That is, the characteristic equation (33) has a pure imaginary $\operatorname{root} \lambda=i \omega_{0}\left(\omega_{0}>0\right)$ if and only if the following statement is true:

$$
\begin{aligned}
\left(i \omega_{0}\right. & \left.+d+\beta v^{*}\right)\left(i \omega_{0}+u\right)\left[i \omega_{0}\left(i \omega_{0}+\delta R_{0}\right)+b\left(R_{0} \delta-a\right)\right] \\
& =i \omega_{0}\left(i \omega_{0}+d\right) u \delta R_{0} e^{i \omega_{0} \tau_{1}} .
\end{aligned}
$$

We claim that the following inequality holds:

$$
\left|i \omega_{0}\left(i \omega_{0}+\delta R_{0}\right)+b\left(R_{0} \delta-a\right)\right|>\left|i \omega_{0}\right| R_{0} \delta .
$$

In fact, we have

$$
\begin{gathered}
\left|i \omega_{0}\left(i \omega_{0}+\delta R_{0}\right)+b\left(R_{0} \delta-a\right)\right|^{2}-\left|i \omega_{0}\right|^{2}\left(R_{0} \delta\right)^{2} \\
=\left[\omega_{0}^{2}-b\left(R_{0} \delta-a\right)\right]^{2} \geq 0 .
\end{gathered}
$$

It follows from $\left|i \omega_{0}+d+\beta v^{*}\right| \geq\left|i \omega_{0}+d\right|,\left|i \omega_{0}+u\right| \geq u$, and the inequality (35) that the modulus of the left-hand side of (34) is greater than the modulus of the right-hand side. This leads to a contradiction. Therefore, we conclude that (33) does not have any root with nonnegative real part. Thus, the CTL-present infection equilibrium $E_{2}$ of system (2) is locally asymptotically stable when $R_{0}>1+\beta k b / u c d$ in the case of $\tau_{2}=0$ and $\tau_{1} \neq 0$.

Theorem 7. If $\tau_{2}=0, \tau_{1} \neq 0$, and $R_{0}>1+\beta k b / u c d$, then the $E_{2}$ is globally asymptotically stable.

Proof. Define a Lyapunov functional

$$
\begin{aligned}
V(t)= & x^{*} g\left(\frac{x}{x^{*}}\right)+e^{m \tau_{1}} y^{*} g\left(\frac{y}{y^{*}}\right)+a_{1} v^{*} g\left(\frac{v}{v^{*}}\right) \\
& +a_{2} z^{*} g\left(\frac{z}{z^{*}}\right)+\beta x^{*} v^{*} \int_{t-\tau_{1}}^{t} g\left(\frac{x(s) v(s)}{x^{*} v^{*}}\right) \mathrm{d} s,
\end{aligned}
$$

where $x^{*}, y^{*}, v^{*}$, and $z^{*}$ satisfy the following equations:

$$
\begin{gathered}
s=d x^{*}+\beta x^{*} v^{*}, \quad \beta e^{-m \tau_{1}} x^{*} v^{*}-a y^{*}-p y^{*} z^{*}=0, \\
k y^{*}=u v^{*}, \quad c y^{*}=b .
\end{gathered}
$$

Calculating the derivative of $V(t)$ along the solution of system (2) and using the similar method with the proof of Theorem 5 , we have

$$
\begin{aligned}
\left.D^{+} V(t)\right|_{(2)} & \\
=d x^{*}\left(2-\frac{x^{*}}{x}-\frac{x}{x^{*}}\right) & \\
-\beta x^{*} v^{*} & {\left[g\left(\frac{x^{*}}{x}\right)+g\left(\frac{y^{*} x\left(t-\tau_{1}\right) v\left(t-\tau_{1}\right)}{y x^{*} v^{*}}\right)\right.} \\
& \left.+g\left(\frac{y v^{*}}{y^{*} v}\right)\right] .
\end{aligned}
$$

Since $2-x^{*} / x-x / x^{*} \leq 0$, it follows that $\left.D^{+} V\right|_{(1.2)} \leq$ 0 for all $x, y, v, z>0$ and $\left.D^{+} V\right|_{(2)}=0$ if and only if $(x(t), y(t), v(t), z(t))=\left(x^{*}, y^{*}, v^{*}, z^{*}\right)$. Then the global attractivity of $E_{2}$ follows from Lyapunov-Lasalle invariance principle [12]. Therefore the CTL-present infection equilibrium $E_{2}$ of system (2) is globally asymptotically stable.

The following discussions focus on the stability of the equilibrium $E_{2}$ in the second case. Let $\tau_{1}=0$ in (32); it follows that that

$$
\begin{aligned}
\lambda^{4}+ & a_{1} \lambda^{3}+a_{2} \lambda^{2}+a_{3} \lambda+a_{4} \\
& +\left(b_{1} \lambda^{3}+b_{2} \lambda^{2}+b_{3} \lambda+b_{4}\right) e^{-\lambda \tau_{2}}=0,
\end{aligned}
$$

where

$$
\begin{aligned}
& a_{1}=b+d+\beta v^{*}+u+\delta R_{0} \\
& a_{2}=b\left(d+\beta v^{*}+u+\delta R_{0}\right)+\left(d+\beta v^{*}\right)\left(u+\delta R_{0}\right), \\
& a_{3}=\delta R_{0} u \beta v^{*}+b\left(d+\beta v^{*}\right)\left(u+\delta R_{0}\right), \\
& a_{4}=b \delta R_{0} u \beta v^{*} \\
& b_{1}=-b \\
& b_{2}=-b\left(d+\beta v^{*}+a+u\right) \\
& b_{3}=b u\left(\delta R_{0}-a\right)-b\left(d+\beta v^{*}\right)(u+a) \\
& b_{4}=b u d \delta R_{0}-a b u d-a b u \beta v^{*}
\end{aligned}
$$

Let

$$
\begin{gathered}
P\left(\lambda, \tau_{2}\right)=\lambda^{4}+a_{1} \lambda^{3}+a_{2} \lambda^{2}+a_{3} \lambda+a_{4}, \\
Q\left(\lambda, \tau_{2}\right)=b_{1} \lambda^{3}+b_{2} \lambda^{2}+b_{3} \lambda+b_{4} .
\end{gathered}
$$

Then we can rewrite (40) as follows:

$$
P\left(\lambda, \tau_{2}\right)+Q\left(\lambda, \tau_{2}\right) e^{-\lambda \tau_{2}}=0 .
$$

Assume that $P\left(\lambda, \tau_{2}\right)$ and $Q\left(\lambda, \tau_{2}\right)$ are analytic functions in the right half-plane $\operatorname{Re} \lambda>-\delta$, where $\delta>0$.

Lemma 8. Consider (43); $P\left(\lambda, \tau_{2}\right)$ and $Q\left(\lambda, \tau_{2}\right)$ satisfy the following conditions: 


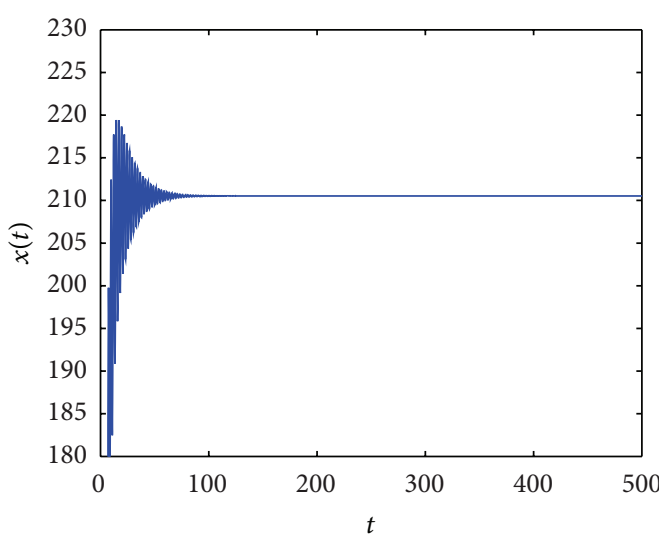

(a)

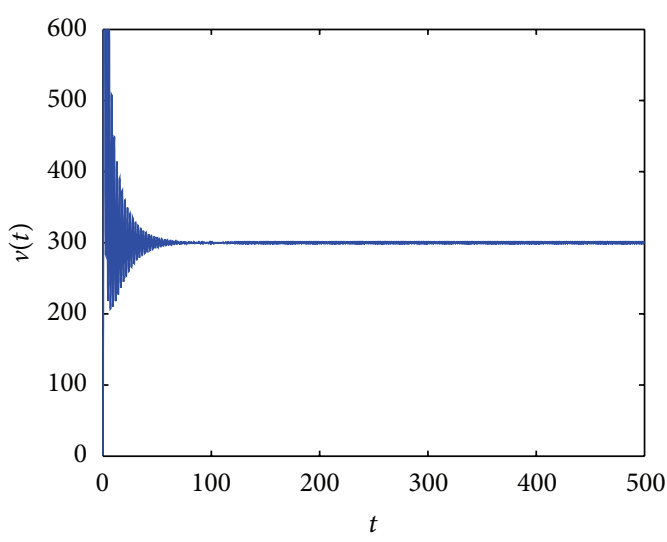

(c)

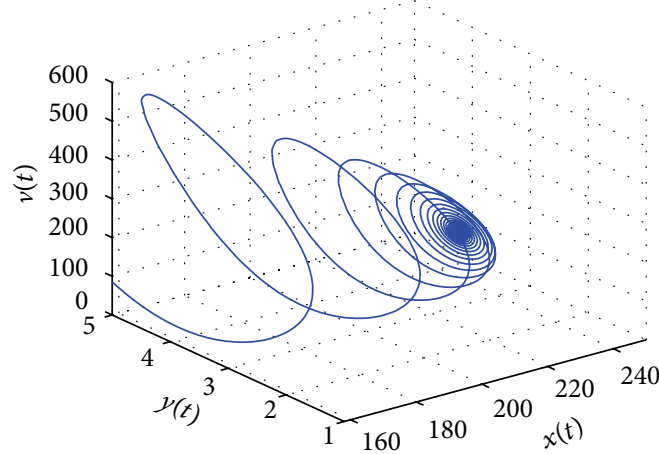

(e)

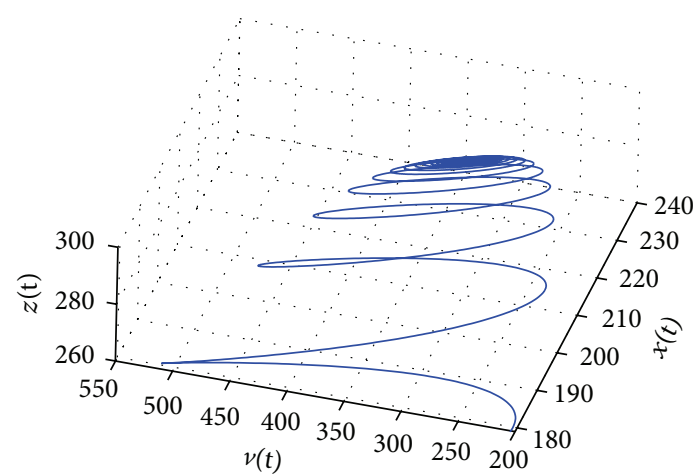

(g)

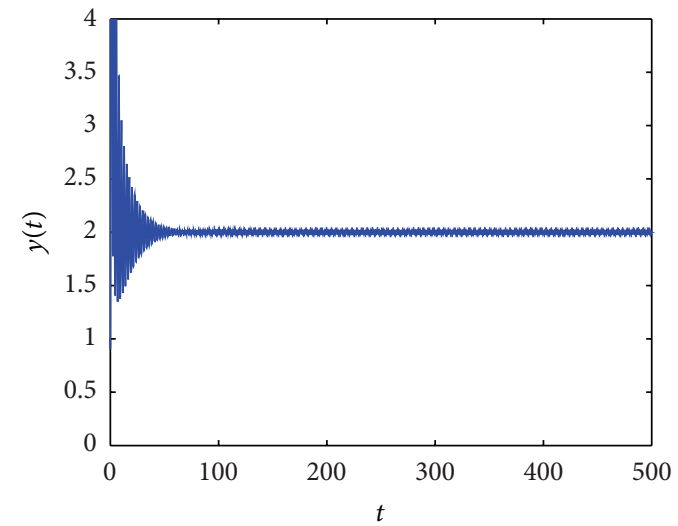

(b)

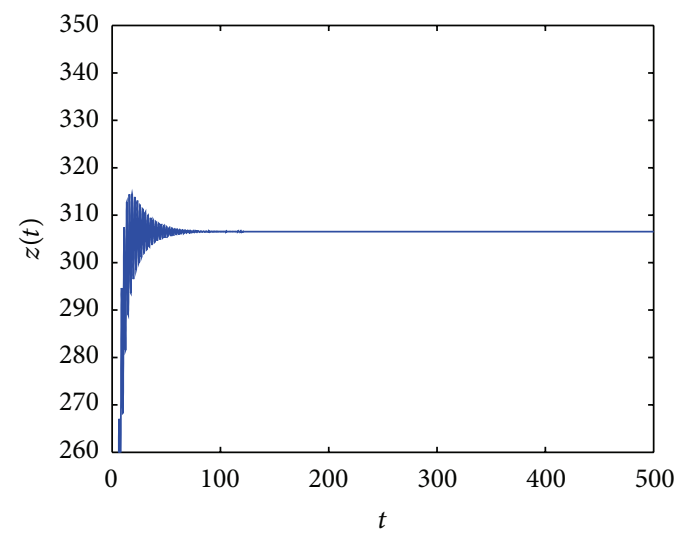

(d)

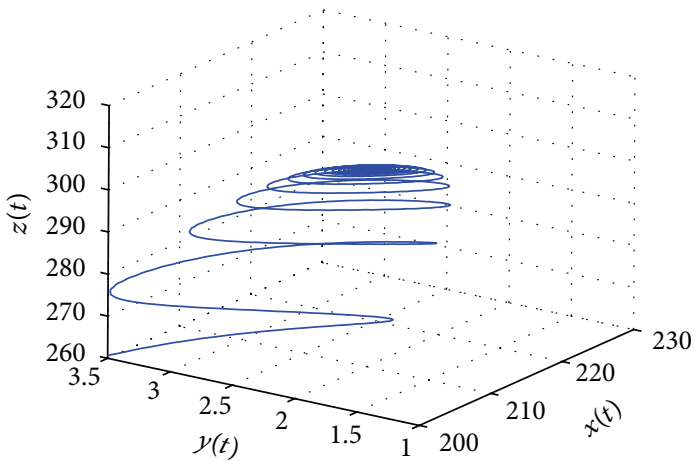

(f)

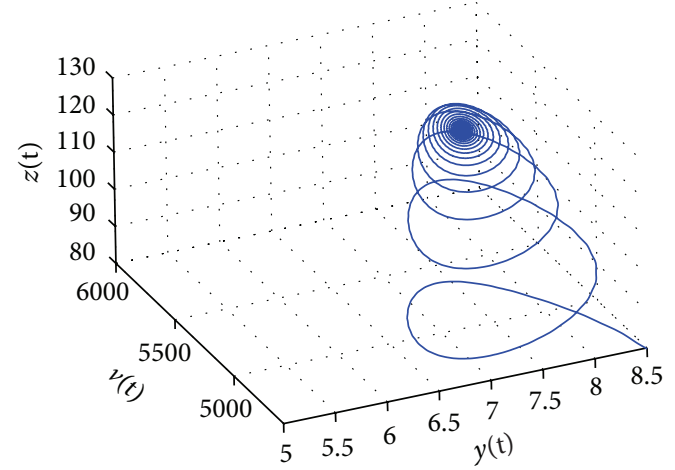

(h)

FIGURE 1: The time histories and the phase trajectories of system (50) before Hopf bifurcation occurs for $\tau_{2}=0.2145$. 


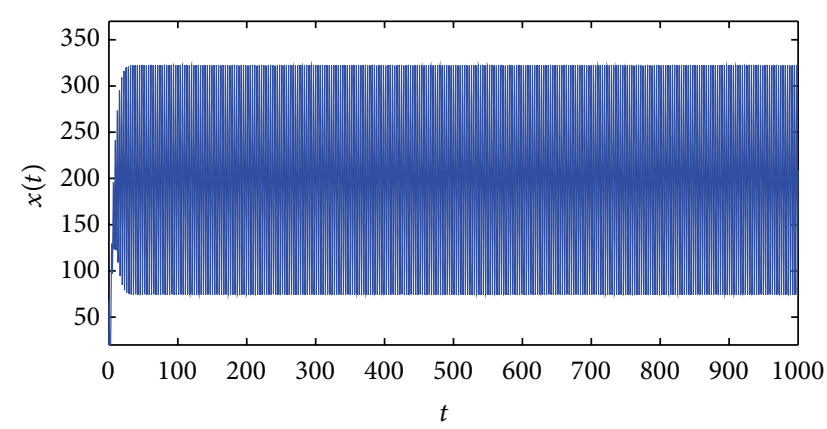

(a)

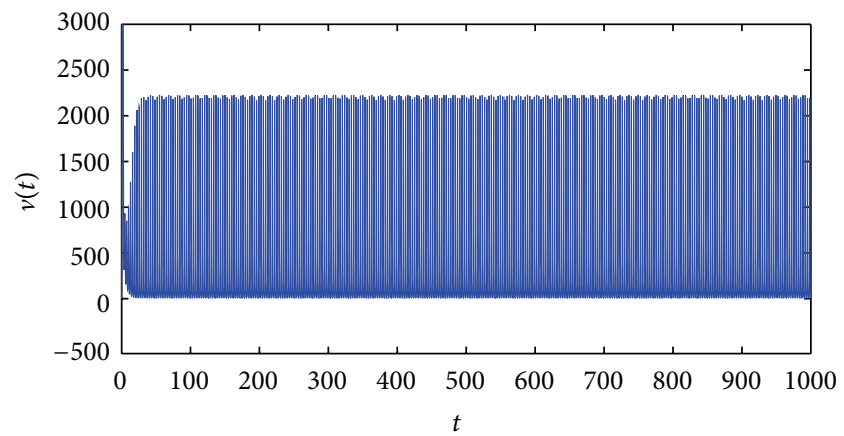

(c)

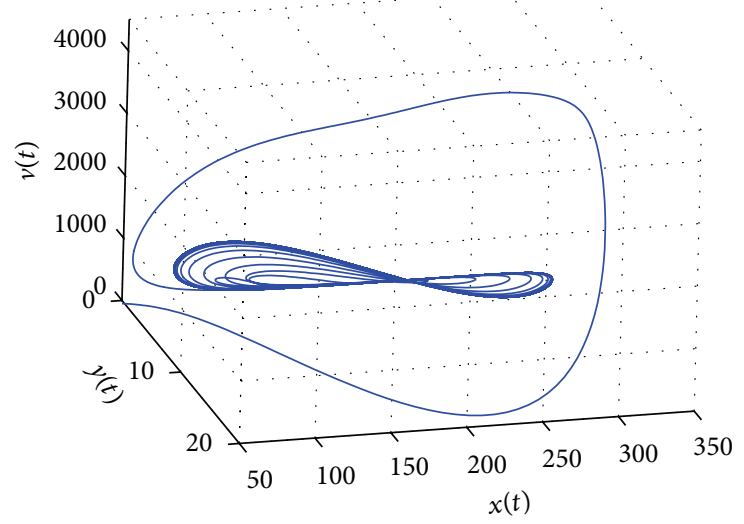

(e)

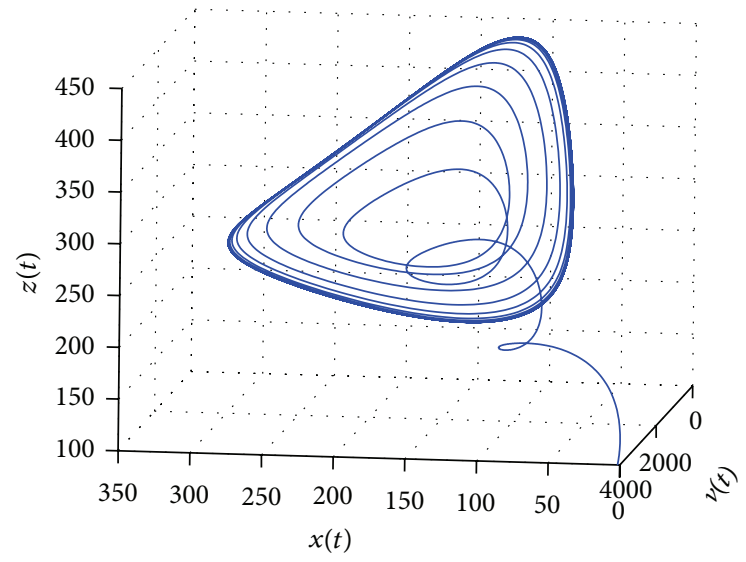

(g)

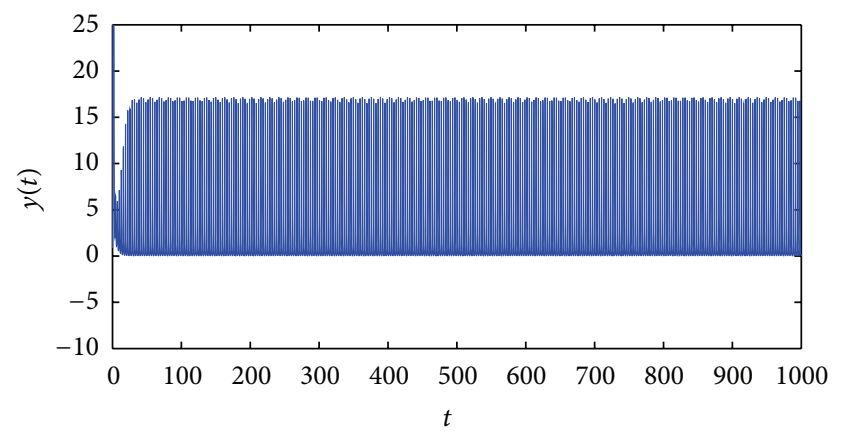

(b)

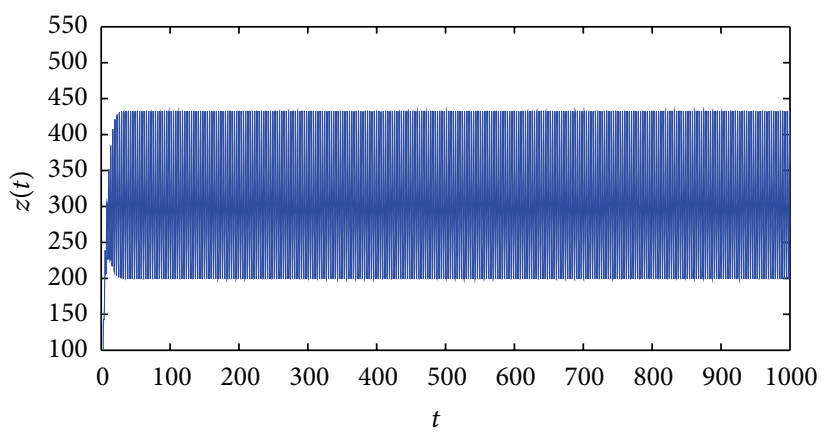

(d)

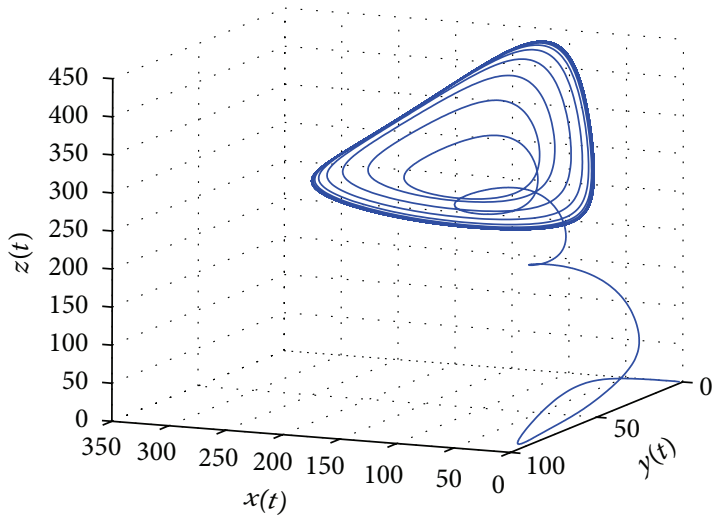

(f)

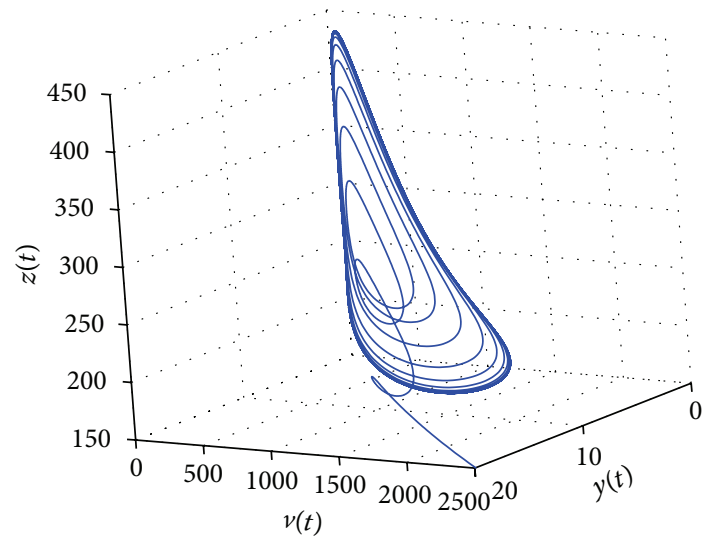

(h)

FIgURE 2: The time histories and the phase trajectories of the system (50) when Hopf bifurcation occurs for $\tau_{2}=0.57$. 


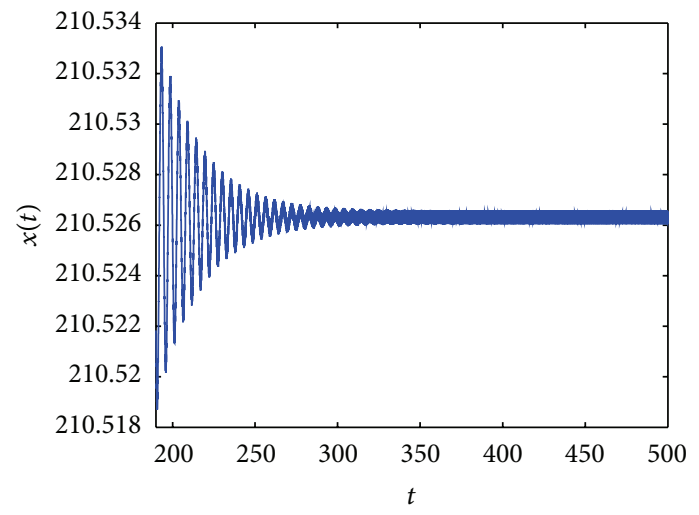

(a)

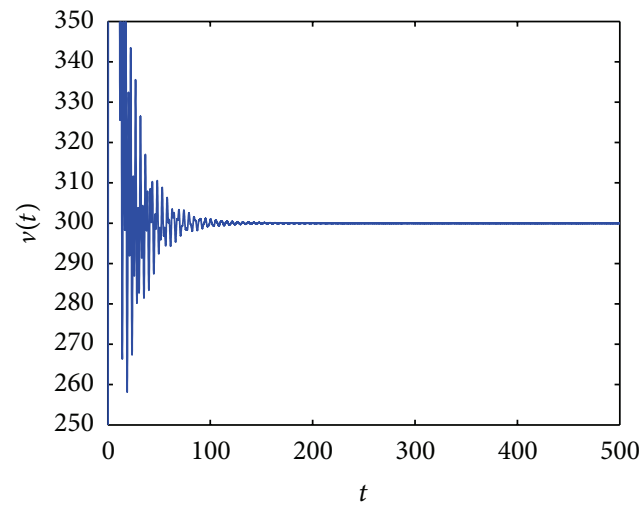

(c)

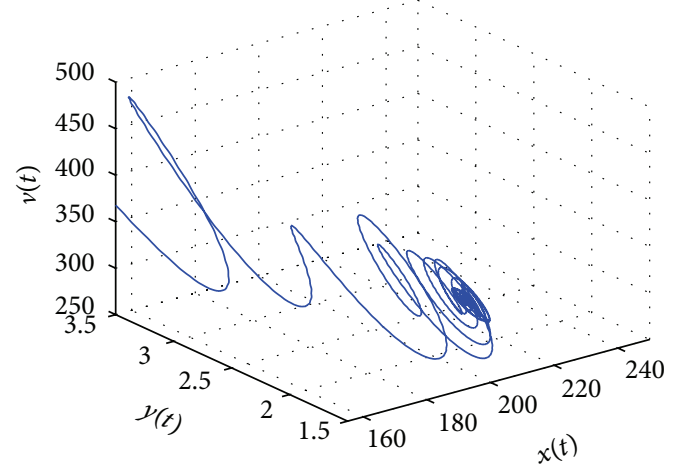

(e)

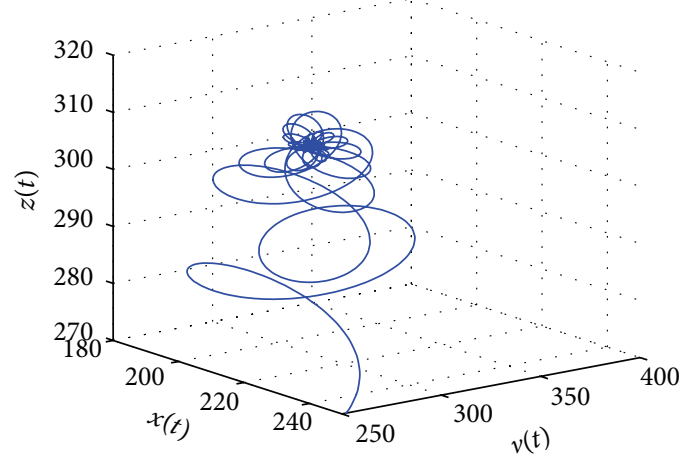

(g)

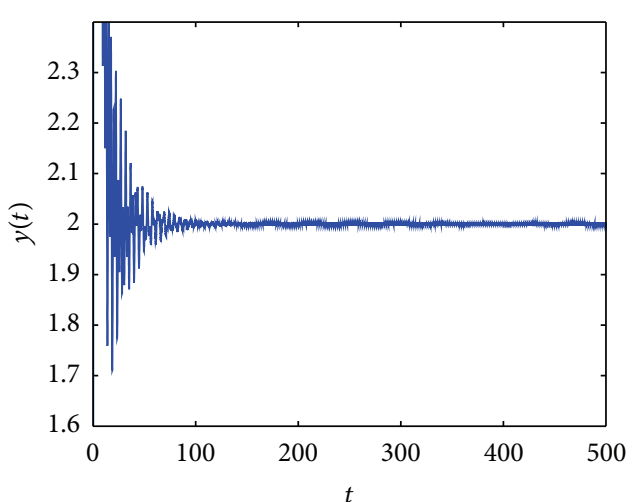

(b)

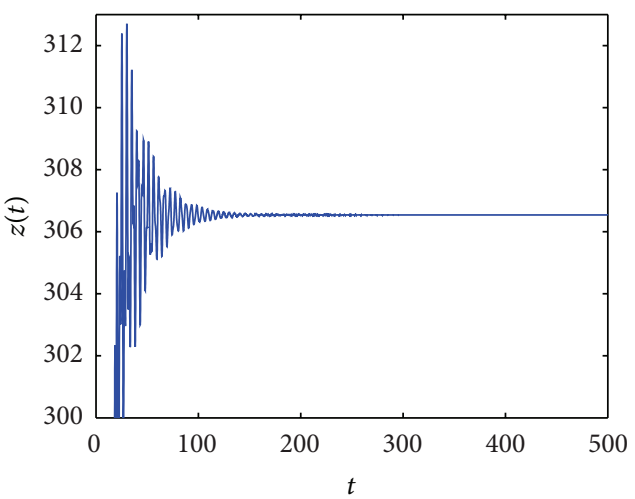

(d)

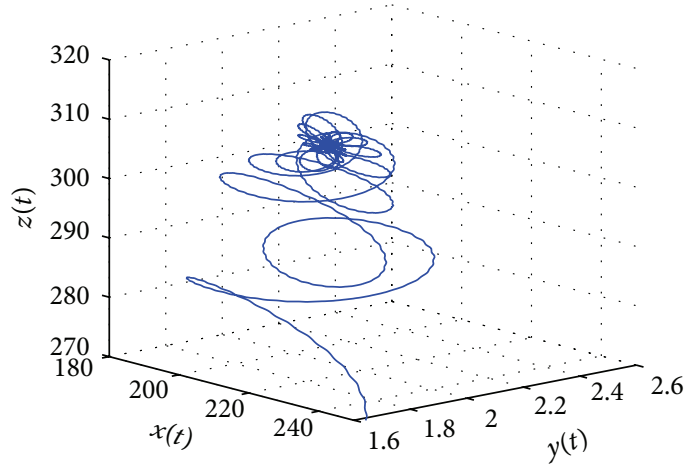

(f)

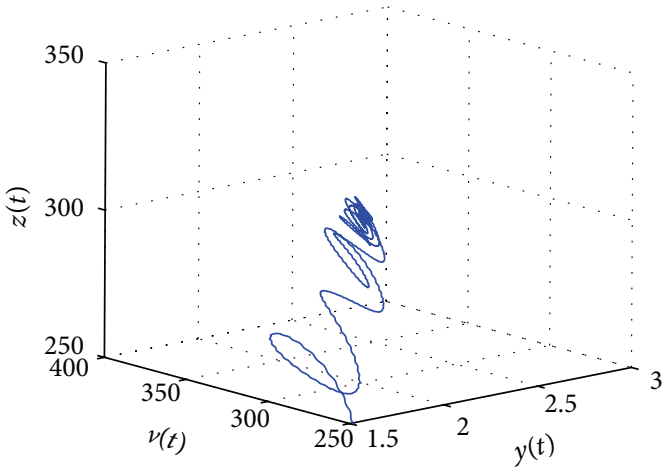

(h)

FIGURE 3: The time histories and the phase trajectories of the system (2) after Hopf bifurcation occurs for $\tau_{2}=2.55$. 
(i) $P\left(0, \tau_{2}\right)+Q\left(0, \tau_{2}\right) \neq 0$;

(ii) $P\left(i w, \tau_{2}\right)+Q\left(i w, \tau_{2}\right) \neq 0$;

(iii) $\lim \sup \left\{\left|Q\left(\lambda, \tau_{2}\right) / P\left(\lambda, \tau_{2}\right)\right|:|\lambda| \rightarrow \infty, \operatorname{Re} \lambda \geq 0\right\}<1$ for any $\tau_{2}$;

(iv) $F(w) \equiv\left|P\left(i w, \tau_{2}\right)\right|^{2}-\left|Q\left(i w, \tau_{2}\right)\right|^{2}$ for real $w$, has at most a finite number of real zeros;

(v) each positive root $w\left(\tau_{2}\right)$ of $F\left(w, \tau_{2}\right)=0$ is continuous and differentiable at $\tau_{2}$ whenever it exists.

Proof. (i) $P\left(0, \tau_{2}\right)+Q\left(0, \tau_{2}\right)=a_{4}+b_{4}=\left(d+\beta v^{*}\right) b u\left(\delta R_{0}-\right.$ a) $\neq 0$. This means $\lambda=0$ is not a characteristic root of (43).

(ii) Consider

$$
\begin{aligned}
P\left(i w, \tau_{2}\right)+Q\left(i w, \tau_{2}\right) \\
=w^{4}-a_{1} w^{3} i-a_{2} w^{2}+a_{3} w i+a_{4} \\
\quad+\left(-b_{1} w^{3} i-b_{2} w^{2}+b_{3} w i+b_{4}\right) \\
=w^{4}-\left(a_{1}+b_{1}\right) w^{3} i-\left(a_{2}+b_{2}\right) w^{2} \\
\quad+\left(a_{3}+b_{3}\right) w i+a_{4}+b_{4} \neq 0 .
\end{aligned}
$$

(iii) Since

$$
\begin{aligned}
& \lim _{|\lambda| \rightarrow \infty}\left|\frac{Q\left(\lambda, \tau_{2}\right)}{P\left(\lambda, \tau_{2}\right)}\right| \\
& =\lim _{|\lambda| \rightarrow \infty}\left|\frac{b_{1} \lambda^{3}+b_{2} \lambda^{2}+b_{3} \lambda+b_{4}}{\lambda^{4}+a_{1} \lambda^{3}+a_{2} \lambda^{2}+a_{3} \lambda+a_{4}}\right|=0,
\end{aligned}
$$

we have $\lim \sup _{|\lambda| \rightarrow \infty, \operatorname{Re} \lambda \geq 0}\left|Q\left(\lambda, \tau_{2}\right) / P\left(\lambda, \tau_{2}\right)\right|<1$ for any $\tau_{2}>0$.

(iv) Since

$$
\begin{aligned}
F\left(w, \tau_{2}\right)= & \left|P\left(i w, \tau_{2}\right)\right|^{2}-\left|Q\left(i w, \tau_{2}\right)\right|^{2} \\
= & w^{8}+\left(-2 a_{2}+a_{1}^{2}-b_{1}^{2}\right) w^{6} \\
& +\left(a_{2}^{2}+2 a_{4}-2 a_{1} a_{3}-b_{2}^{2}+2 b_{1} b_{3}\right) w^{4} \\
& +\left(-2 a_{2} a_{4}+a_{3}^{2}+2 b_{2} b_{4}-b_{3}^{2}\right) w^{2}+a_{4}^{2}-b_{4}^{2} .
\end{aligned}
$$

It is obvious that property (iv) is satisfied.

(v) The last assertion is valid because $F\left(w, \tau_{2}\right)$ is a quadratic polynomial in $w^{2}$ and the fact that $a_{i}, b_{j}(i, j=$ $1,2,3,4)$ are all continuous functions of $\tau_{2}$. This completes the proof.

Let $z=w^{2}$ and denote $p=-2 a_{2}+a_{1}^{2}-b_{1}^{2}, q=a_{2}^{2}+2 a_{4}-$ $2 a_{1} a_{3}-b_{2}^{2}+2 b_{1} b_{3}, r=-2 a_{2} a_{4}+a_{3}^{2}+2 b_{2} b_{4}-b_{3}^{2}$, and $u_{0}=a_{4}^{2}-b_{4}^{2}$; then (46) becomes

$$
F(z)=z^{4}+p z^{3}+q z^{2}+r z+u_{0}=0 .
$$

On the distribution of positive roots of (46), we have the following results.

Lemma 9. If $u_{0}<0$, then (46) has at least one positive root.
Proof. Since $F(w)=w^{8}+p w^{6}+q w^{4}+r w^{2}+u_{0}=0$ and $F(0)=u_{0}<0, \lim _{w \rightarrow+\infty} F(w)=+\infty$. Hence, there exists at least an $w_{0}>0$ such that $F\left(w_{0}\right)=0$.

In the following, we will analyze the case $u_{0} \geq 0$. Let $z=$ $y-p / 4$; then (47) can be rewritten as

$$
y^{4}+p_{1} y^{2}+q_{1} y+r_{1}=0
$$

where $p_{1}=-3 p^{2} / 8+q, q_{1}=p^{3} / 8-p q / 2+r, r_{1}=-3 p^{4} / 256+$ $\left(p^{2} / 16\right) q-p r / 4+u_{0}$. If $q_{1}=0$, then it is easy to obtain the four roots of (48) as follows

$$
\begin{array}{ll}
y_{1}=\sqrt{\frac{-p_{1}+\sqrt{\Delta_{0}}}{2}}, \quad y_{2}=-\sqrt{\frac{-p_{1}+\sqrt{\Delta_{0}}}{2}}, \\
y_{3}=\sqrt{\frac{-p_{1}-\sqrt{\Delta_{0}}}{2}}, \quad y_{4}=-\sqrt{\frac{-p_{1}-\sqrt{\Delta_{0}}}{2}},
\end{array}
$$

where $\Delta_{0}=p_{1}^{2}-4 r_{1}$. Thus $z_{i}=y_{i}-(p / 4)(i=1,2,3,4)$ are the roots of (47). Then we have the following result.

Lemma 10. Suppose that $u_{0} \geq 0$ and $q_{1}=0$.

(i) If $\Delta_{0}<0$, then (47) has no positive real roots.

(ii) If $\Delta_{0} \geq 0, p_{1}>0$ and $r_{1}>0$, then (47) has no positive real roots.

Based on Theorem 4.1 in [12] and Lemmas 8-10, we have the following results.

Theorem 11. (i) If $u_{0} \geq 0, q_{1}=0$;
(a) $\Delta_{0}<0$
(b) $\Delta_{0} \geq 0, p_{1}>0$, and $r_{1}>0$,

the equation $F(w)=0$ has no positive roots. Then system (2) is stable for all $\tau_{2} \geq 0$.

(ii) If $u_{0}<0$, the equation $F(w)=0$ has at least one positive root and each positive root is simple; then stability switches may occur as $\tau_{2}$ increases. That is, there exists a positive number $\tau_{2}^{*}$ such that (2) is unstable for all $\tau_{2}>\tau_{2}^{*}$. As $\tau_{2}$ varies from 0 to $\tau_{2}^{*}$, at most a finite number of stability switches may occur.

\section{Numerical Simulations}

In the previous sections, we studied dynamical behaviors of the system (2) and obtained some important results. In this section, we perform a numerical analysis of the model based on Theorem 11. 
Example 12. By corresponding to system (2), we consider the following system:

$$
\begin{gathered}
\frac{d x}{d t}=160-0.16 x(t)-0.002 x(t) v(t), \\
\frac{d y}{d t}=0.002 e^{-2 \tau_{1}} x\left(t-\tau_{1}\right) v\left(t-\tau_{1}\right) \\
-1.85 y(t)-0.2 y(t) z(t), \\
\frac{d v}{d t}=1200 y(t)-8 v(t), \\
\frac{d z}{d t}=0.2 y\left(t-\tau_{2}\right) z\left(t-\tau_{2}\right)-0.4 z(t),
\end{gathered}
$$

where $\tau_{1}=0$. By direct calculation we can get CTLpresent infection equilibrium $E_{2}=(210.5263,2.0000$, $300.0000,306.5395)$ for system (50).

From the parameters given in system (50) and the values of $\tau_{2}$, we can see that there are two critical values of the delay $\tau_{2}$, denoted by $\tau_{2}^{*}$ and $\tau_{2}^{* *}, \tau_{2}^{*} \approx 0.2611, \tau_{2}^{* *} \approx 2.4276$. Simple numerical simulations show that the CTL-present infection equilibrium of system (50) is globally asymptotically stable for $\tau_{2} \in\left[0, \tau_{2}^{*}\right)$ (see Figure 1 ). In this case, we take $\tau_{2}=$ $0.2145<\tau_{2}^{*}$. The above CTL-present infection equilibrium $E_{2}$ of system (50) is unstable for $\tau_{2} \in\left(\tau_{2}^{*}, \tau_{2}^{* *}\right)$ (see Figure 2). In this case, we take $\tau_{2}=0.57>\tau_{2}^{*}$. The above CTLpresent infection equilibrium $E_{2}$ of system (50) is globally asymptotically stable for $\tau_{2}>\tau_{2}^{* *}$ (see Figure 3 ). In this case, we take $\tau_{2}=2.55>\tau_{2}^{* *}$. From above analysis we can conclude that Hopf bifurcation occurs when $\tau_{2} \in\left(\tau_{2}^{*}, \tau_{2}^{* *}\right)$.

\section{Conclusions}

In this paper, we have discussed HIV infection model with intracellular delay and CTL-response delay. We assume that the production of CTLs depends on the infected cells and CTL cells for some important biological meanings. Dynamical analysis of system (2) shows that intracellular delay $\tau_{1}$ and immune delay $\tau_{2}$ play different roles in the stability of the equilibrium. The results show that when $R_{0}<1$, the infection-free equilibrium is globally asymptotically stable, which means that the viruses are cleared and immune is not active. When $1<R_{0}<1+\beta k b / u c d$, the CTL-absent infection equilibrium exists and is globally asymptotically stable, which means that the CTL immune response would not be activated and viral infection becomes vanished. When $R_{0}>1+\beta k b / u c d$ and $\tau_{2}=0$, the CTL-present infection equilibrium is globally asymptotically stable. Actually, the intracellular delay does not affect the stability of the system. When $R_{0}>1+\beta k b / u c d$ and $\tau_{1}=0$, system (2) may undergo a stability switch.

\section{Disclosure}

The authors declare that they have no financial or personal relationships with other people or organizations that can inappropriately influence their work; there is no professional or other personal interest of any nature or kind in any product, service and/or company that could be construed as influencing the position presented in, or the paper.

\section{Acknowledgment}

This work was supported by the National Natural Science Foundation of China (Grants nos. 11261056, 11261058, and 11271312).

\section{References}

[1] R. V. Culshaw and S. Ruan, "A delay-differential equation model of HIV infection of $\mathrm{CD}^{+}$T-cells," Mathematical Biosciences, vol. 165, no. 1, pp. 27-39, 2000.

[2] R. V. Culshaw, S. Ruan, and G. Webb, "A mathematical model of cell-to-cell spread of HIV-1 that includes a time delay," Journal of Mathematical Biology, vol. 46, no. 5, pp. 425-444, 2003.

[3] P. W. Nelson and A. S. Perelson, "Mathematical analysis of delay differential equation models of HIV-1 infection," Mathematical Biosciences, vol. 179, no. 1, pp. 73-94, 2002.

[4] P. W. Nelson, J. D. Murray, and A. S. Perelson, "A model of HIV-1 pathogenesis that includes an intracellular delay," Mathematical Biosciences, vol. 163, no. 2, pp. 201-215, 2000.

[5] H. Zhu and X. Zou, "Impact of delays in cell infection and virus production on HIV-1 dynamics," Mathematical Medicine and Biology, vol. 25, no. 2, pp. 99-112, 2008.

[6] A. A. Canabarro, I. M. Gléria, and M. L. Lyra, "Periodic solutions and chaos in a non-linear model for the delayed cellular immune response," Physica A, vol. 342, no. 1-2, pp. 234241, 2004.

[7] K. Wang, W. Wang, H. Pang, and X. Liu, "Complex dynamic behavior in a viral model with delayed immune response," Physica D, vol. 226, no. 2, pp. 197-208, 2007.

[8] K. A. Pawelek, S. Liu, F. Pahlevani, and L. Rong, "A model of HIV-1 infection with two time delays: mathematical analysis and comparison with patient data," Mathematical Biosciences, vol. 235, no. 1, pp. 98-109, 2012.

[9] M. Y. Li and H. Shu, "Global dynamics of an in-host viral model with intracellular delay," Bulletin of Mathematical Biology, vol. 72, no. 6, pp. 1492-1505, 2010.

[10] H. Zhu and X. Zou, "Dynamics of a HIV-1 infection model with cell-mediated immune response and intracellular delay," Discrete and Continuous Dynamical Systems B, vol. 12, no. 2, pp. 511-524, 2009.

[11] C. A. Janeway, P. Travers, M. Walport, and M. J. Schlomchik, Immunobiology, Garland Science Publishing, 6th edition, 2005.

[12] Y. Kuang, Delay Differential Equations with Applications in Population Dynamics, Academic Press, San Diego, Calif, USA, 1993.

[13] J. K. Hale, Theory of Functional Differential Equations, Springer, New York, NY, USA, 1997.

[14] X. Wang, A. Elaiw, and X. Song, "Global properties of a delayed HIV infection model with CTL immune response," Applied Mathematics and Computation, vol. 218, no. 18, pp. 9405-9414, 2012.

[15] H. Zhu, Y. Luo, and M. Chen, "Stability and Hopf bifurcation of a HIV infection model with CTL-response delay," Computers and Mathematics with Applications, vol. 62, no. 8, pp. 3091-3102, 2011. 
[16] E. Beretta and Y. Kuang, "Geometric stability switch criteria in delay differential systems with delay dependent parameters," SIAM Journal on Mathematical Analysis, vol. 33, no. 5, pp. 11441165, 2002.

[17] M. A. Nowak and C. R. M. Bangham, "Population dynamics of immune responses to persistent viruses," Science, vol. 272, no. 5258, pp. 74-79, 1996.

[18] J. K. Hale and S. Lunel, Introduction to Functional Differential Equations, Springer, New York, NY, USA, 1993.

[19] T. Zhang, J. Liu, and Z. Teng, "Stability of Hopf bifurcation of a delayed SIRS epidemic model with stage structure," Nonlinear Analysis: Real World Applications, vol. 11, no. 1, pp. 293-306, 2010.

[20] X. Song, X. Zhou, and X. Zhao, "Properties of stability and Hopf bifurcation for a HIV infection model with time delay," Applied Mathematical Modelling, vol. 34, no. 6, pp. 1511-1523, 2010. 


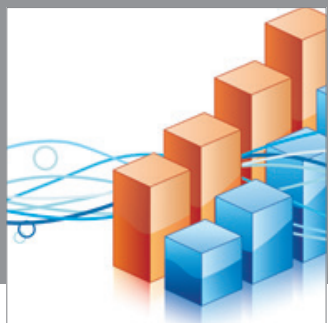

Advances in

Operations Research

mansans

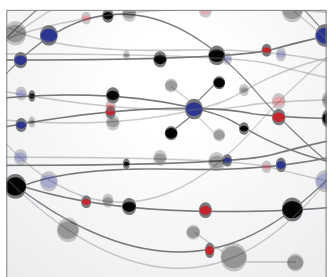

The Scientific World Journal
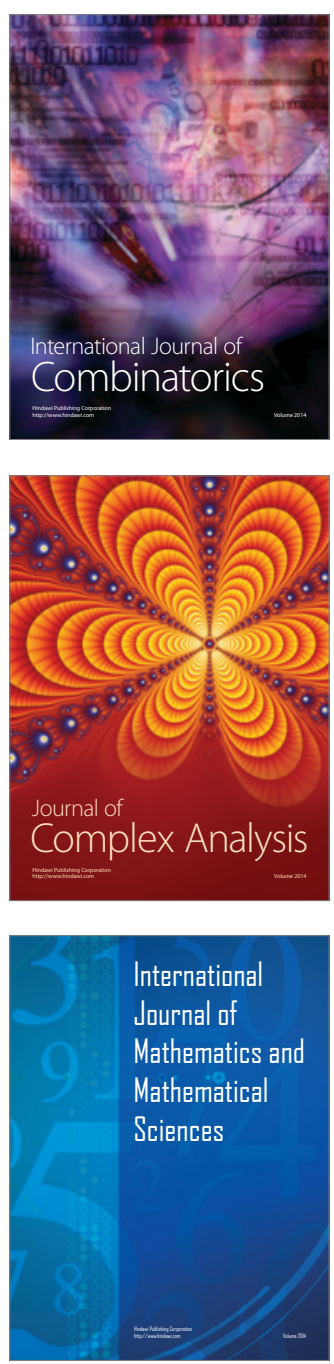
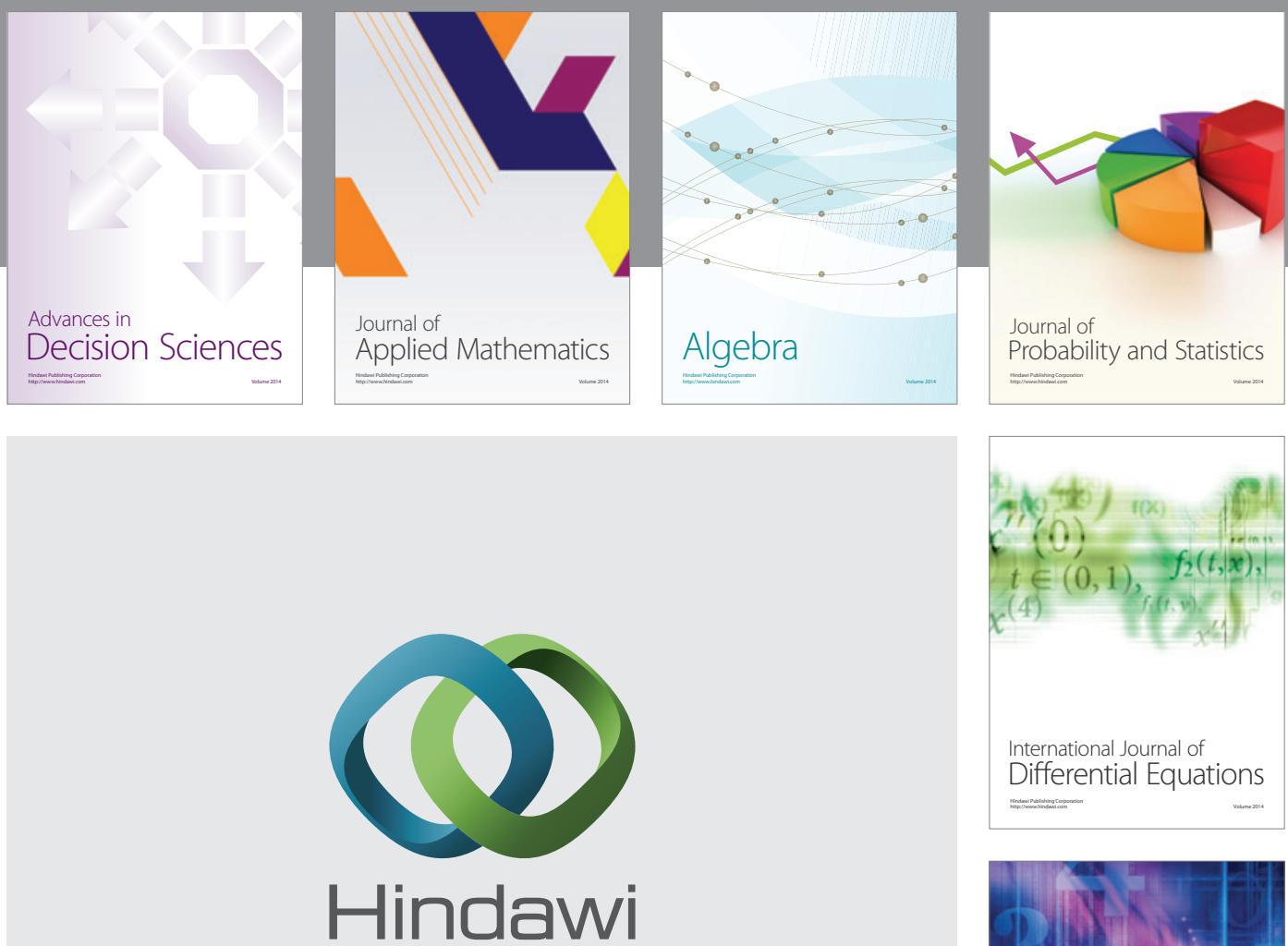

Submit your manuscripts at http://www.hindawi.com
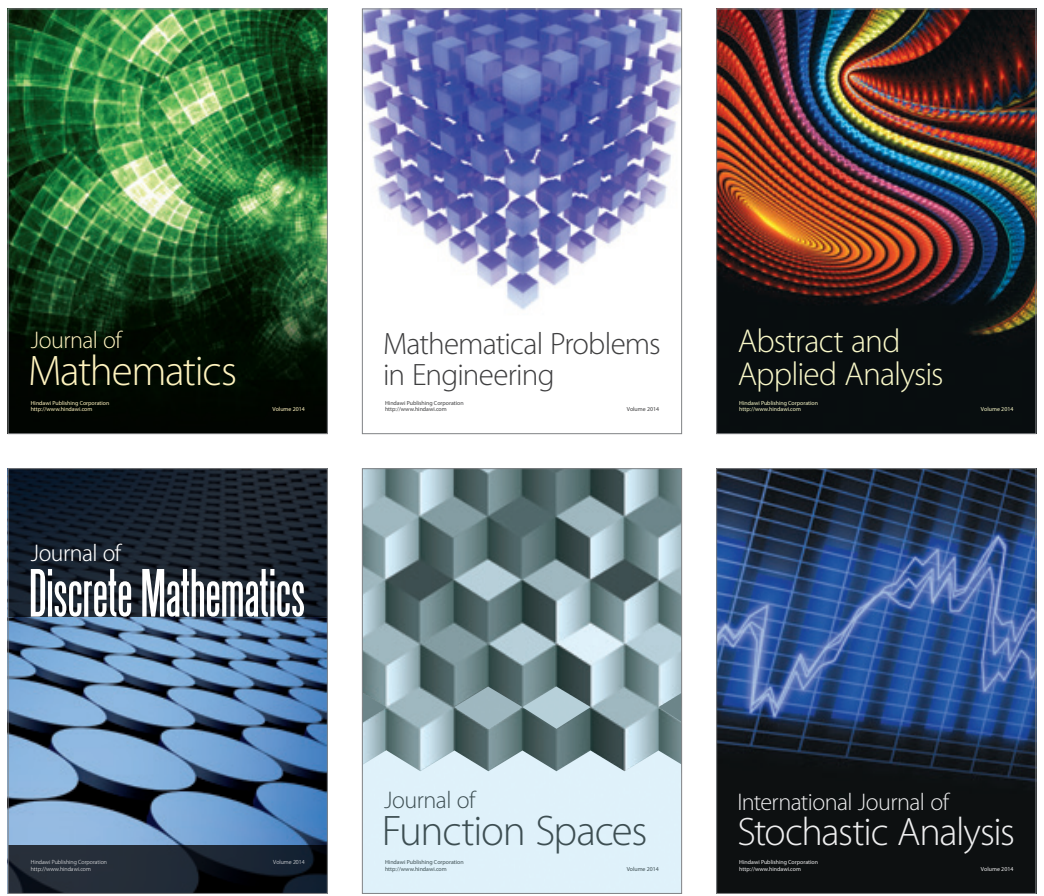

Journal of

Function Spaces

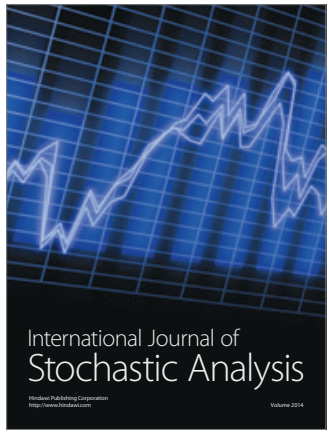

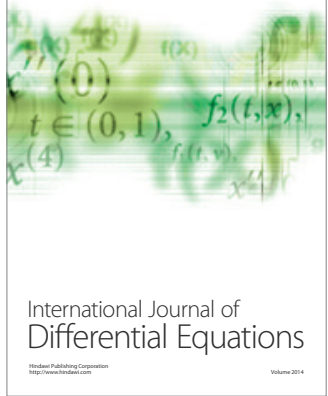
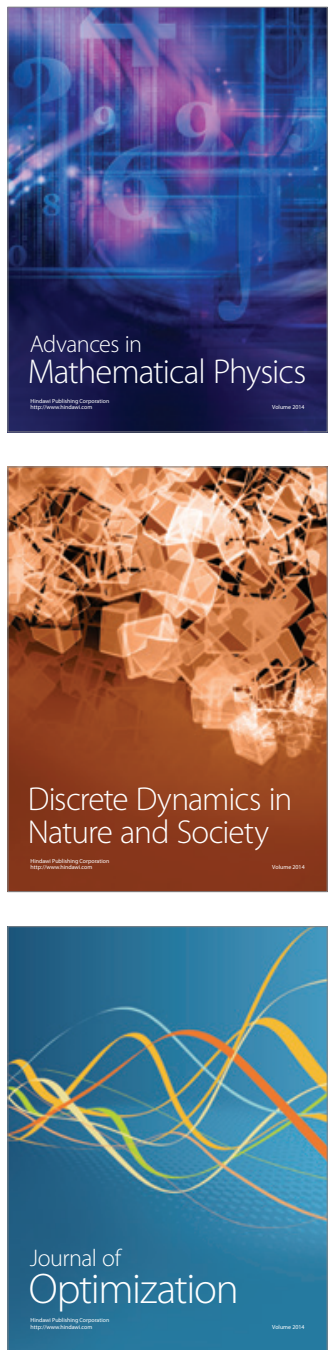